\title{
Deserción estudiantil: incidencia de factores institucionales relacionados con los procesos de admisión
}

\section{Omar Alberto Tapasco Alzate}

orcid.org/oooo-0002-9751-6737

Universidad de Caldas, Colombia

omar.tapasco@ucaldas.edu.co

\section{David Osorio García}

orcid.org/00oo-0002-9944-364X

Universidad de Caldas, Colombia

david.osorio@ucaldas.edu.co
Francisco Javier Ruiz Ortega

orcid.org/000o-0003-1592-5535

Universidad de Caldas, Colombia

francisco.ruiz@ucaldas.edu.co

\section{Resumen}

La presente investigación estudia la incidencia que distintas variables institucionales relacionadas con el proceso de admisión tienen sobre el riesgo de deserción estudiantil, asi como el efecto que tienen las ponderaciones asignadas a las áreas evaluadas en el puntaje de admisión en correlación con el desempeño académico. La población objetivo fueron los estudiantes matriculados en la Universidad de Caldas para las cohortes 2010-2012. Para ello se hace uso de modelos logísticos multivariados y de técnicas de optimización. Los modelos obtenidos arrojan que algunas variables del proceso de admisión, susceptibles de ser intervenidas institucionalmente, como puntaje de admisión, ingreso por segunda opción, programas de cupos especiales y costo de matrícula, tienen una incidencia estadisticamente significativa en el riesgo de deserción, influencia que varía entre facultades. Como conclusión, en la perspectiva de atenuar la deserción estudiantil y de incrementar el poder predictivo del desempeño futuro del estudiante, se deberian contemplar acciones tales como el repensar el ingreso por segunda opción, el sostener las políticas de ingreso por méritos académicos y de acción afirmativa y el modificar las ponderaciones actualmente asignadas a las áreas evaluadas en el proceso de admisión.

\section{Palabras clave (fuente: tesauro de la UnEsco).}

Admisión en la universidad; condiciones de admisión; análisis multivariado; criterios de ingreso; deserción escolar; selección de estudiantes.

Recepción: 02/06/2018 | Envío a pares: 11/08/2018 | Aceptación por pares: 07/09/2018 | Aprobación: 09/03/2019 


\title{
Dropping Out: Incidence of Institutional Factors Related to Admission Processes
}

\begin{abstract}
This research studies the incidence of different institutional variables related to the admission process on dropout risk and the effect of weights assigned to the areas tested on the admission score in correlation with academic performance. Target population was students enrolled in the Universidad de Caldas for 2010-2012 cohorts. For this, multivariate logistic models and optimization techniques were used. Models obtained show that some variables of the admission process-subject to institutional intervention-, such as admission score, second option admission, special availability programs and tuition costs, have a statistically significant incidence on dropout risk that varies among schools. As a conclusion, in order to minimize dropping out and increase the predictive power of student's future performance, actions such as rethinking second option admission, sustaining academic achievement admission and affirmative action policies, and modifying the weights currently assigned to the areas tested in the admission process should be considered.
\end{abstract}

\section{Keywords (Source: UnEsco Thesaurus)}

Admission criteria; admission requirements; multivariate analysis; student admission; dropping out; student selection. 


\section{Evasão estudantil: incidência de fatores institucionais relacionados aos processos de admissão}

\section{Resumo}

Esta pesquisa estuda a incidência que diferentes variáveis institucionais relacionadas com o processo de admissão têm sobre o risco de evasão estudantil, bem como o efeito que têm as ponderações designadas às áreas avaliadas na pontuação de admissão em correlação com o desempenho acadêmico. A população-alvo foi de estudantes matriculados na Universidad de Caldas, Colômbia, para as coortes 2010-2012. Para isso, faz-se uso de modelos logísticos multivariados e de técnicas de otimização. Os modelos obtidos demonstram que algumas variáveis do processo de admissão, suscetiveis de serem intervindas institucionalmente, como pontuação de admissão, ingresso por segunda opção, programas de vagas especiais e custo de matrícula têm uma incidência estatisticamente significativa no risco de evasão, influência que varia entre faculdades. Como conclusão, na espera de diminuir a evasão estudantil e de aumentar o poder preditivo do desempenho futuro do estudante, deveriam ser contempladas ações como repensar o ingresso por segunda opção, sustentar as políticas de ingresso por méritos acadêmicos e por ação afirmativa, e modificar as ponderações atualmente designadas às áreas avaliadas no processo de admissão.

\section{Palavras-chave (Fonte: Tesauro da Unesco)}

Admissão na universidade; condições de admissão; análise multivariada; critérios de entrada; evasão escolar; seleção de estudantes. 


\section{Introducción}

El proceso de admisión, además de predecir el desempeño académico estudiantil en la universidad, debería proveer mecanismos de selección que identifiquen el potencial para tener éxito en los estudiantes de orígenes relativamente desfavorecidos, evitando de esta forma la discriminación por factores étnicos, raciales, etarios o de género, conducentes a reducir el riesgo de la deserción de los sistemas educativos. Sobre esta última, se conoce que es una problemática global con afectaciones que trascienden el plano individual y repercuten en el desarrollo de las sociedades en las que los desertores se insertan (Esteban, Bernardo, Tuero, Cerezo y Núñez, 2016).

Al respecto, algunas estimaciones de costos de deserción arrojan que para el año 2009 los derivados de la deserción universitaria en Colombia fueron de $\$ 778 \mathrm{mil}$ millones de pesos, aproximadamente US\$380 millones de dólares, a precios de la época (MEN, 2010), mientras que para las universidades españolas el fenómeno de deserción se cuantificó en $€ \$ 3.000$ millones de euros anuales, lo que supone un importante desaprovechamiento de los recursos públicos, que se incrementa si se contemplan los costos asumidos por los estudiantes y sus familias (Esteban, Bernardo, Tuero, Cervero y Casanova, 2017).

Hay consenso en aceptar que la tasa de abandono es un indicador de baja calidad, pues se entiende que la universidad no proporcionó los medios necesarios para que los estudiantes, que lo intentaron, obtuvieran el título esperado. Razones que justifican, en los escenarios educativos nacionales e internacionales, la creciente preocupación por la incidencia de aspectos sociales y académicos en los procesos de admisión a la universidad y en el fenómeno multicausal de la deserción, y que sustentan la posibilidad de suponer que la realización de un buen diagnóstico del problema es una inversión de la institución, en la idea de que todo ello, acompañado de la aplicación de medidas preventivas y correctivas establecidas en función del mismo, conducirá a un gran ahorro para la sociedad (Esteban, Bernardo, Tuero, Cervero y Casanova, 2017).

En esta misma línea de trabajo, se plantea que una manera de reducir la deserción es la aplicación de medidas anticipadas al ingreso a la institución de educación superior, para que el alumnado tome decisiones vocacionales acordes a sus perfiles (Cabrera, Tomás, González y Álvarez, 2006). De igual manera se proponen acciones orientadas a la obtención de un sistema de alertas tempranas en la detección de los perfiles de riesgo de la deserción estudiantil, de tal forma que las instituciones de educación superior pudiesen adelantar acciones de ajuste y acompañamiento en procura de menguar dicha problemática.

En particular, Colombia vive una coyuntura especial en el ámbito educativo, ya que, de un lado, las políticas gubernamentales conducentes a favorecer la inclusión educativa han permitido la ampliación de la cobertura, con incrementos en la tasa neta de matrículas del 48\% para el año 2013, comparada con el 28\% logrado en el año 2004 (MEN, 2016). No obstante, de otro lado, estos indicadores de cobertura no han sido ajenos a incrementos graduales y sostenidos en las tasas de deserción (Barragán y Patiño, 2013). Una explicación razonable surge del hecho de que dicho incremento en la participación en educación superior está asociado a la incorporación de estudiantes pertenecientes a grupos socioeconómicos que años atrás prácticamente no la tenían y a que un alto número de instituciones no ha reaccionado oportunamente a esta situación, en la búsqueda de formas de adaptación a este nuevo conjunto de grupos sociales con funciones y demandas específicas (Fonseca y García, 2016).

\section{Generalidades de la deserción}

$\mathrm{El}$ abandono estudiantil, como lo afirma el Ministerio de Educación colombiano, es un "problema dual, ya que no solo es importante conocer sus causas, sino la manera de disminuirla, lo cual significa 
aumentar la retención estudiantil" (MEN, 2009, p. 136), característica que lo ratifica como un problema muy complejo y difícil de combatir, pese a ser un fenómeno altamente estudiado (De Vries, León, Romero y Hernández, 2011). Varias investigaciones han demostrado que este fenómeno obedece a factores personales, institucionales y académicos (Tinto, 1993; Bean, 1990; Cabrera, Tomás, González y Álvarez, 1992; Cabrera, Pérez y López, 2015). Para Khuong (2014), el abandono está condicionado por un conjunto de variables vinculadas con los antecedentes y características sociales, psicológicas, institucionales y aspectos individuales de los estudiantes, entre otras. Por su parte, Esteban, Bernardo, Tuero, Cerezo y Núñez (2016) afirman que el fenómeno hace referencia a cinco factores: individuales, académicos, económicos, institucionales y culturales, pero hacen énfasis en que el rendimiento académico previo es una variable con alta incidencia en la permanencia del alumno universitario.

Para Torres (2012), los modelos explicativos de la deserción se pueden agrupar según el enfoque: psicológico, sociológico, económico, organizacional e interaccionista, complementario e integrado. Las diferencias entre los enfoques se encuentran en el peso que tienen las variables individuales o estructurales para explicar la deserción. Para dicha investigación, la propuesta presentada inicialmente por Tinto (1975), que incluye variables estructurales de los estudiantes, compromisos y cualidades institucionales, y el modelo de Weidman (1989), citado por Donoso y Schiefelbein (2007, p. 20), que articula "factores sicológicos y socio estructurales [...] [e involucra] la elección de la carrera", se convierten en una guía teórica para la comprensión de la deserción estudiantil en los niveles individuales y organizativos de la universidad.

De otro lado, diversidad de informes y estudios realizados sobre la temática ubican a la dimensión académica como el factor central, asociado al capital cultural y académico con el que ingresan los estudiantes a la educación superior, seguido por factores socioeconómicos y factores institucionales, ligados a políticas y normativas, infraestructura, relaciones con el profesorado y con los demás estudiantes, calidad de los programas, programas de acompañamiento para incentivar la permanencia y los de orientación profesional (Barragán y Patiño, 2013). Entre estas últimas, se resaltan aquellas variables propias del ambiente institucional que pueden modificarse para favorecer las trayectorias estudiantiles, por lo que el llamado a las instituciones es a conocer al estudiante de primer año para gestionar programas de apoyo y estrategias dirigidas a estimular su compromiso y persistencia (Silva, 2011).

Entre los factores académicos, algunas de las deficiencias detectadas en los estudiantes que ingresan a la universidad, manifestadas como carencias en competencias comunicativas, analíticas, de sintesis y conceptualización, son atribuibles a las inequidades en los sistemas educativos, que en la educación media afectan de manera marcada a estudiantes de bajos ingresos provenientes de municipios pobres (Barragán y Patiño, 2013). En esta perspectiva, Sánchez, Quirós, Reverón y Rodríguez (2002) ratifican la necesidad de comprender en profundidad e intervenir las desigualdades sociales y educativas de los estudiantes que egresan de las instituciones de educación media, y centran su análisis en las diferencias educativas de los padres, pues es un fenómeno que indiscutiblemente repercute en la desigualdad social y en la posibilidad de competir por el acceso de sus hijos a los centros de educación superior y que, al ingresar a los centros educativos, incide notablemente en el rendimiento académico y en la posibilidad de desertar del sistema educativo.

\section{Uso de pruebas estandarizadas en los procesos de admisión}

Se ha discutido extensamente sobre el rol de las pruebas estandarizadas en los procesos de admisión a las universidades, polémicas avivadas por el reducido aporte de las puntuaciones de dichas pruebas en la predicción de los resultados de quienes 
asisten a la universidad (Tapasco, Ruiz y Osorio, 2016) y su significativo impacto negativo en los estudiantes de contextos desfavorecidos, para quienes tales puntuaciones actúan como una barrera que dificulta su ingreso a la educación superior (Geiser, 2016). Específicamente para el caso colombiano, los puntajes de las pruebas Saber 11 muestran una correlación directa con el nivel socioeconómico del que proviene el estudiante, por lo que los estudiantes de estratos bajos ven limitadas sus posibilidades de acceder a una educación superior. Esta situación podría llevar a pensar que la educación no está sirviendo como un instrumento para favorecer a la movilidad social, sino reproduciendo los privilegios sociales y económicos de los estratos altos (Sánchez y Otero, 2012).

De otro lado, el resultado de las pruebas estandarizadas como Saber 11 ha servido para caracterizar el riesgo de deserción de los estudiantes universitarios, dado que los estudiantes con peor clasificación presentan una mayor tasa de deserción y los de mejor clasificación menores tasas (MEN, 2008), con el agravante de que en los últimos años el porcentaje de estudiantes matriculados con baja calificación ha aumentado, lo que genera preocupación, al tener una mayor cantidad de estudiantes ubicados en rangos de alto riesgo de deserción (CEDE, 2014).

\section{Implementación de acciones afirmativas en los procesos de admisión}

Se conoce, además, que son particularmente críticos los registros de mortalidad y promedios académicos observados en los estudiantes de comunidades indígenas y negritudes, que se convierten de hecho en los más vulnerables, en términos de permanencia en la universidad (Tonconi, 2010). Por ello la deserción universitaria ha sido tratada ampliamente como una de las manifestaciones atribuidas a la desigualdad social, en lo que toca a las oportunidades de acceso, a lo riesgos de deserción y a las tasas de titulación, en donde los grupos sociales menos favorecidos reflejan situaciones alejadas de la igualdad de derechos y oportunidades (Escanés, Herrero, Merlino y Ayllón, 2014).

En el contexto británico, Hoare y Johnston (2011) manifiestan que muchas instituciones ya han comenzado a gestionar el acceso a la universidad de estudiantes provenientes de entornos desfavorecidos mediante la introducción de programas de participación cada vez más amplios. Los autores cuestionan la existencia de muy pocas investigaciones en este campo que exploren el desempeño de los estudiantes favorecidos por tales programas. Otros estudios evidencian las políticas que intentan mejorar la equidad en el acceso de la educación y la permanencia de población vulnerable en la institución de educación superior del Caribe colombiano (Morón, Pedrozo y Torres, 2013) y concluyen que han sido eficientes en términos del número de personas beneficiadas para el acceso, pero no conducentes en la reducción de la deserción.

Dentro de las políticas para disminuir la brecha de las desigualdades sociales está el crédito educativo, puesto en marcha por el Instituto Colombiano de Crédito Educativo y Estudios Técnicos en el Exterior (Icetex), pero el trabajo de Blanco (2014) hace énfasis en que la política estatal y el mercado han cedido terreno a la democratización de la educación. En este mismo sentido, Gómez y Celis (2009) argumentan que los créditos educativos terminan endeudando a los estudiantes de condiciones más desfavorables y, a su vez, las acciones afirmativas que realizan las universidades solo se encaminan a asuntos económicos, desconociendo, en ambas situaciones, la historia formativa de los estudiantes, la cual no les permite tener las competencias necesarias para un rendimiento adecuado y mejorar las tasas de permanencia y graduación. A pesar de esto, Melguizo, Sánchez y Jaime (2011), a partir de un análisis de regresión logística, establecen que la ayuda financiera reduce la tasa de deserción, sobre todo en estudiantes que inician la carrera universitaria.

Por otra parte, Navarrete, Candia y Puchi (2013), a través de una investigación con estudiantes mapu- 
ches en Chile, identifican que los grupos étnicos que ingresan a la universidad tienen mayor probabilidad de deserción en el primer año de estudios, causada por condiciones socioeconómicas limitadas y baja calidad de la educación previa. Por lo tanto, existe una relación directa con la deserción entre las condiciones socialmente desfavorables y la etnicidad. En este mismo sentido, Castellanos, Correa, y Loaiza (2006) afirman que los grupos étnicos, indígenas y negritudes que ingresaron a la Universidad de Caldas, entre 2001 y 2004, tenían condiciones sociales desfavorables, dado que pertenecían en su mayoría a estratos socioeconómicos 1 y 2 y egresaban de colegios públicos. En sus propias palabras: "la política de acceso preferencial mediante cupos especiales no solo tiene un trasfondo de carácter ético, sustentado en la exclusión histórica a la que han estado sometidas estas comunidades, sino a la exclusión económica y política a la cual están expuestos hoy" (2006, p. 141).

\section{Aspectos vocacionales en la selección del programa de interés}

Se plantea que la vocación está relacionada con los factores motivacionales que llevan a un individuo a elegir una carrera universitaria. Para Ruiz y Rodríguez, identificar por qué se toma la decisión de ingresar a una u otra carrera es relevante, por al menos tres razones: primero, porque es un reconocimiento de que la elección "si bien es un acto individual, como decisión, es una acción compleja y multifactorial que involucra la relación de elementos culturales, políticos, económicos, sociales y familiares" (2011, p. 13); segundo, porque los aspectos motivacionales implícitos en esta decisión afectan el encuentro pedagógico entre estudiantes y docentes, en el aula de clases; y tercero, porque esos aspectos motivacionales demandarán del estudiante, según sea su interés por la carrera que cursa, un compromiso que puede facilitar u obstaculizar su rendimiento académico.

Varias son las investigaciones y autores que valoran la relación que existe entre componentes como el rendimiento académico, la deserción, el nivel de satisfacción del estudiante con su carrera y el orden de escogencia de la misma. Por ejemplo, para Garbanzo (2007), la elección de los estudios según el interés de los estudiantes -categoría, según ella, traslapada con los intereses motivacionales o personales del estudiante- es un indicador relevante del rendimiento del estudiante universitario, que servirá también para explicar el trasfondo de los procesos educativos de calidad de las instituciones y para monitorear y ejecutar recursos estatales proyectados al mejoramiento de los mismos procesos. En esta misma línea de investigación, el estudio de Marín, Infante y Troyano (2000), realizado con estudiantes desertores del sector educativo universitario, evidencia una importante relación entre el fracaso académico y la elección de los estudios universitarios, independientemente del nivel de inteligencia del estudiante, por lo que el fracaso no puede explicarse basándose en forma exclusiva en el déficit intelectual o cognoscitivo, sino que deben considerarse otros aspectos condicionantes de índole actitudinal.

\section{Asignación de ponderaciones a los puntajes de admisión}

Algunos estudios señalan que uno de los aspectos de manejo discrecional por las instituciones que puede contribuir a la mejora predictiva del desempeño académico es la asignación de las ponderaciones a las pruebas que componen los procesos de admisión. Hacen así el llamado a la revisión de dichas ponderaciones en procura de incrementar la capacidad predictiva de los procesos de selección universitaria (Vergara y Peredo, 2017). Por su lado, Makransky, Havmose, Vang, Andersen y Nielsen (2016) proponen ir más allá de las pruebas cognoscitivas para incrementar la validez predictiva de los procesos de admisión y sugieren la realización de múltiples minientrevistas, cuyo objetivo sea la valoración de habilidades no cognitivas, tales como la autoeficacia, la motivación intrínseca y el pensamiento crítico. 
Dados los referentes expuestos, la presente investigación se propuso indagar el efecto de distintas variables de tipo institucional, relacionadas con el proceso de admisión y, por ende, susceptibles de ser intervenidas, sobre la deserción estudiantil, además de otras variables de tipo individual, que han mostrado asociación con las tasas de deserción. En igual sentido, se presenta una propuesta de evaluación para las ponderaciones asignadas al puntaje de admisión acudiendo a procesos de optimización de las mismas, dirigidas a incrementar la correlación con el desempeño académico del estudiante.

\section{Materiales y métodos}

El estudio realizado es de carácter retrospectivo, dado que recolecta datos de las cohortes 2010 a 2012 de los estudiantes de la Universidad de Caldas, Manizales, en Colombia, proporcionados por la Oficina de Registro Académico de la Universidad. El total de ingresos registrados en los programas de pregrado para los años referenciados que contenían información completa fue de 6.451 estudiantes, distribuidos por facultades: Ciencias Agropecuarias $11 \%$, Artes y Humanidades $20 \%$, Ciencias Exactas y Naturales 14,8\%, Ingenierías 10,9\%, Ciencias Jurídicas y Sociales $28,1 \%$ y Ciencias de la Salud $15,2 \%$; por género en $51,7 \%$ hombres y $48,3 \%$ mujeres. Con respecto a su procedencia, $49 \%$ eran del departamento de Caldas, $17,6 \%$ provenían de otros departamentos y $33,4 \%$ no registraba información.

Los datos de deserción fueron obtenidos del Sistema para la Prevención de la Deserción de la Educación Superior (SPADIES), plataforma que se implementó en Colombia en 2002 como herramienta para hacer seguimiento a las cifras de deserción. En afinidad con el tratamiento de los datos en el SPADIES, se define la deserción desde una perspectiva institucional, considerando como desertor aquel estudiante que abandona la institución educativa durante dos períodos consecutivos (CEDE, 2014). Con los datos suministrados por las instituciones a SPADIES, se identifican y se ponderan los comportamientos, las causas, las variables y los riesgos asociados (Barragán y Patiño, 2013).

Específicamente para este estudio, dentro de los factores institucionales relacionados con el proceso de admisión, se incluyeron las variables ponderación de las puntuaciones en las pruebas de Estado, la opción de admisión, el tipo de admisión y el costo de la matrícula. Para el procesamiento de los datos se recurrió a la selección de las variables de interés en hojas de Excel, que posteriormente fueron migradas al R-3.4.3, para la obtención de los modelos descriptivos y el cálculo de optimización de las ponderaciones y al Statistical Package for the Social Sciences, versión 22, para la obtención de los estadísticos descriptivos univariados y bivariados.

Dado que la variable respuesta es de tipo dicotómico, se plantea la utilización de un modelo logístico o un modelo probit. Para la selección del modelo más adecuado se acude al test de Hausman, el cual contrasta los coeficientes de ambos modelos, en la búsqueda de aquellos con mayor eficiencia estadística. Para tal fin, se calcula dicha estadística con un código en $\mathrm{R}$, que involucra una forma cuadrática entre los coeficientes y sus matrices de varianzas y covarianzas, el cual sigue una distribución chi cuadrado (Green, 2012). El valor resultante de dicho estadístico es 101,46 ( $P=0.000)$, indicando con ello que el modelo más eficiente es el logístico. Por lo tanto, se recurre a un modelo de regresión logística, asumiendo valores de cero (o), si no es desertor, y de uno (1), si lo es. Las distintas variables contempladas en el presente estudio, su escala de medición, sus categorías o rangos y los estadísticos descriptivos básicos se recopilaron en la Tabla 1.

De otra parte, la Universidad tiene contemplado en su normativa de admisiones el asignar ponderaciones a las áreas temáticas que componen las pruebas del Instituto Colombiano para el Fomento de la Educación Superior (Icfes) o pruebas de Estado, en la búsqueda de una mayor alineación entre la aptitud evidenciada en las pruebas y el perfil 
Tabla 1. Descriptivos de las variables utilizadas para el grupo total y los subgrupos de deserción*

\begin{tabular}{|c|c|c|c|c|}
\hline \multicolumn{5}{|c|}{ Variables de escala de razón } \\
\hline \multirow[t]{2}{*}{ Variables } & \multirow[t]{2}{*}{ Niveles } & \multirow{2}{*}{$\begin{array}{c}\text { Global } \\
(\mathrm{N}=6421) \\
\text { Media } \pm \text { D.E. }\end{array}$} & $\begin{array}{c}\text { No desertor } \\
(n=3746)\end{array}$ & $\begin{array}{l}\text { Desertor } \\
(\mathrm{n}=2675)\end{array}$ \\
\hline & & & \multicolumn{2}{|c|}{ Media \pm E.E.M } \\
\hline \multirow[t]{2}{*}{ Icfes ponderado } & \multirow{2}{*}{$\begin{array}{c}\text { Puntaje de admisión, ponderado según } \\
\text { normativa institucional }\end{array}$} & \multirow{2}{*}{$\begin{array}{c}55,3 \\
\pm 8.0\end{array}$} & $\begin{array}{r}56,2 \\
\pm 0,13 \\
\end{array}$ & $\begin{aligned} & 54 \\
& \pm 0,15 \\
&\end{aligned}$ \\
\hline & & & \multicolumn{2}{|c|}{$P=0.000$} \\
\hline \multirow{2}{*}{ Costos de matrícula } & \multirow{2}{*}{$\begin{array}{l}\text { Costo primera matrícula, } \\
\text { en salarios mínimos mensuales }\end{array}$} & \multirow{2}{*}{$\begin{array}{l}0,6 \\
\pm 1,1\end{array}$} & $\begin{array}{r}0,63 \\
\pm 0,02 \\
\end{array}$ & $\begin{array}{c}0,47 \\
\pm 0,02\end{array}$ \\
\hline & & & \multicolumn{2}{|c|}{$P=0.000$} \\
\hline \multirow[t]{2}{*}{ Edad } & \multirow[t]{2}{*}{ Edad al momento del ingreso a la universidad } & \multirow{2}{*}{$\begin{array}{r}19,4 \\
\pm 3,2\end{array}$} & $\begin{aligned} & 19,3 \\
\pm & 0,05\end{aligned}$ & $\begin{array}{r}19,7 \\
\pm 0,06 \\
\end{array}$ \\
\hline & & & \multicolumn{2}{|c|}{$P=0.000$} \\
\hline \multicolumn{5}{|c|}{ Variables de escala nominal } \\
\hline \multirow[t]{2}{*}{ Variables } & \multirow{2}{*}{$\begin{array}{l}\text { Niveles } \\
\text { (Codificación) }\end{array}$} & \multirow{2}{*}{$\begin{array}{c}\text { Global } \\
(\mathrm{N}=6421) \\
n(\%)\end{array}$} & $\begin{array}{c}\text { No desertor } \\
(n=3746)\end{array}$ & $\begin{array}{l}\text { Desertor } \\
(\mathrm{n}=2675)\end{array}$ \\
\hline & & & \multicolumn{2}{|c|}{$\mathrm{n}(\%)^{* *}$} \\
\hline \multirow{2}{*}{ Sexo } & \multirow{2}{*}{$\begin{array}{l}\text { Femenino (1) } \\
\text { Masculino (o) }\end{array}$} & \multirow{2}{*}{$\begin{array}{c}3100(48,3) \\
3321(51,7)\end{array}$} & $1944(51,9)$ & $1156(43,2)$ \\
\hline & & & \multicolumn{2}{|c|}{$P=0.000$} \\
\hline \multirow{2}{*}{ Tipo de colegio } & \multirow{2}{*}{$\begin{array}{l}\text { Oficial (o) } \\
\text { Privado (1) }\end{array}$} & \multirow{2}{*}{$\begin{array}{l}5019(78,2) \\
1402(21,8)\end{array}$} & $2933(78,3)$ & 2086 (78.0) \\
\hline & & & $P=$ & 381 \\
\hline Onción de admición & Segunda opción (o) & $336(5,2)$ & $113(3.0)$ & $223(8.3)$ \\
\hline 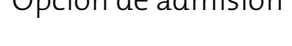 & Primera opción (1) & $6085(94,8)$ & $P=$ & Doo \\
\hline & 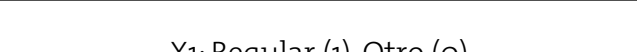 & & $3301(88,1)$ & $2407(90)$ \\
\hline Tipo de admisión & AI: Reguld (1), Utro (O) & R: $5708(88,9)$ & $P=$ & 010 \\
\hline$\left(X_{1}, X_{2}\right)$ & X2.Méritos académicos (1) Otro (0) & A.A: $470(7,3)$ & $168(4,5)$ & $75(2,8)$ \\
\hline & 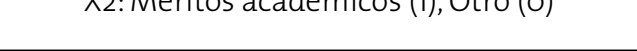 & & $P=$ & o०o \\
\hline & Variable respuesta & Niveles & & criptivos \\
\hline & Desorción & No desertor & & $6(58,3)$ \\
\hline & Deserciom & Desertor & & $75(41,7)$ \\
\hline
\end{tabular}

* El valor P corresponde a la comparación entre medias o proporciones entre los subgrupos, según sea el caso." Para las pruebas de comparación de proporciones la categoría de interés es la primera enunciada.

Fuente: elaboración propia.

del programa. Es así como, mientras para el programa de Biología se les da mayor valoración a los puntajes obtenidos en las áreas de Lenguaje, Matemáticas y Biología, para el de Geología se les da mayor peso a los puntajes registrados en las áreas Química, Matemática y Física, por dar un ejemplo. Lo anterior responde a una lógica de orientación vocacional y de rendimiento aptitudinal evidente. El cuestionamiento que nos hacemos va dirigido al valor de dichas ponderaciones, ya que, aunque fueron los colectivos docentes de los programas respectivos los que las asignaron por concertación, en ningún momento se ha evaluado la pertinencia de tales ponderaciones. 
Para valorar la eficacia de las ponderaciones asignadas, se propone aplicar un procedimiento de optimización conducente a encontrar las ponderaciones óptimas que, a la luz de los resultados presentados en la cohorte bajo estudio, permitirían una mejor selección de los estudiantes admitidos y de esta manera contrastarlos con las ponderaciones existentes, de forma tal que sirvan de insumo a los colectivos respectivos para el replanteamiento de dichas ponderaciones, según sea el caso. Para tal propósito, se plantea maximizar el coeficiente de correlación entre el puntaje de admisión ponderado y el promedio de notas obtenido por el estudiante en la carrera, mediante el paquete DEoptim del R, el cual usa heurísticas de optimización basadas en algoritmos genéticos y estrategias evolutivas para encontrar el óptimo global de funciones de valor real y de parámetros de valor real y no requiere que la función sea continua o diferenciable (Mullen, Ardia, Gil, Windovern y Cline, 2011).

\section{Resultados}

Como se referenció en la Tabla 1, la deserción para las cohortes 2010-2012 en la Universidad de Caldas fue de $41,7 \%$, y aunque representa un valor alto de abandono estudiantil, se ubica por debajo de los referentes nacionales, ya que para el 2015 se registró una tasa de deserción global en instituciones universitarias de 46,1\% según datos del MEN (2016). Para entrar en el análisis de los factores asociados a la problemática de deserción, se recurrió inicialmente a la obtención de estadísticos bivariados de las variables analizadas. En primera instancia, se observa en la Figura 1 la distribución del puntaje de admisión para las dos poblaciones de interés, registrándose que los desertores ingresaron con un puntaje promedio inferior a los no desertores en un poco más de dos puntos por debajo.

Para hacerles seguimiento a las variables de tipo categórico, se recurrió a la obtención de los porcentajes de deserción condicionales para cada categoría de respuesta, con su respectiva significancia

\section{Figura 1. Distribución de los puntajes de admisión para estudiantes desertores y no desertores}

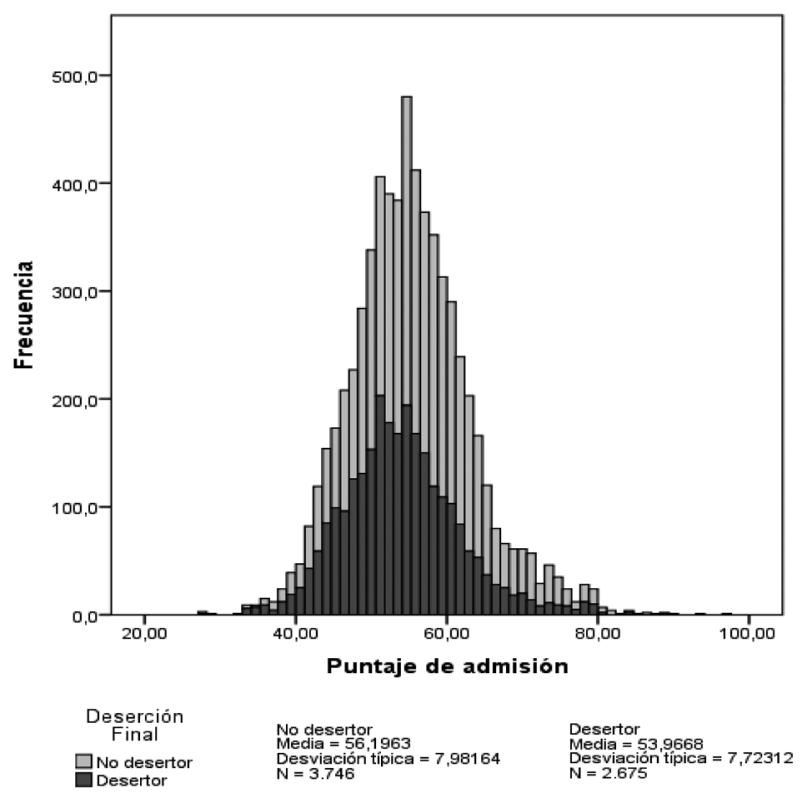

Fuente: elaboración propia.

de la prueba de independencia, tal como se muestra en la Tabla 2.

En primera instancia, se puede observar que el hecho de haber ingresado al programa mediante la modalidad de segunda opción está asociado a un alto riesgo de deserción $(66,4 \%, P=0.000)$, es decir, dos de cada tres estudiantes que ingresaron de esta manera desertaron, y aunque, como se presentó en la Tabla 1, son representativamente pocos, ya que solo el 5,2\% ingresó al programa como segunda opción, estar bajo esta clasificación se convierte en un indicador de alarma para la institución. Mientras que para el tipo de admisión también se presentaron diferencias significativas $(P=0,002)$ y, como era de esperarse, aquellos estudiantes que ingresaron a universidad por su historial académico destacado tuvieron una menor deserción (30,9\%). De otro lado, los resultados por género son coherentes con las estadísticas gubernamentales, en donde se expresa que el hecho de ser hombre está asociado a un 
Tabla 2. Frecuencias condicionales de deserción según categorías de las variables de interés y sus significancias estadísticas

\begin{tabular}{|c|c|c|c|}
\hline Variables de admisión & Categorias de respuesta & Porcentaje de deserción & Valor $\mathrm{P}$ \\
\hline \multirow{2}{*}{ Opción de admisión } & Primera opción & $40,3 \%$ & 0,0 \\
\hline & Segunda opción & $66,4 \%$ & \\
\hline \multirow{3}{*}{ Tipo de admisión } & Acción afirmativa & $41,1 \%$ & 0,002 \\
\hline & Regular & $42,2 \%$ & \\
\hline & Méritos académicos & $30,9 \%$ & \\
\hline Variables del estudiante & Niveles & Porcentaje de deserción & Valor $\mathrm{P}$ \\
\hline \multirow{2}{*}{ Sexo } & Masculino & $45,7 \%$ & \multirow{2}{*}{0,0} \\
\hline & Femenino & $37,3 \%$ & \\
\hline \multirow{2}{*}{ Tipo de colegio } & Oficial & $41,6 \%$ & \multirow{2}{*}{0,664} \\
\hline & Privado & $42,0 \%$ & \\
\hline
\end{tabular}

Fuente: elaboración propia.

riesgo mayor de deserción comparado con el de las mujeres $(P=0.000)$ (MEN, 2008). Y la comparación del tipo de colegio del cual egresan los estudiantes genera diferencias no significativas estadísticamente $(P=0,664)$.

Con el propósito de ampliar la perspectiva de análisis, se recurrió a la obtención de un modelo logístico multivariado, el cual permite, al contrario del análisis bivariado expuesto anteriormente, evidenciar las posibles interdependencias entre las variables explicativas involucradas. Los resultados obtenidos al ajustar el modelo logístico a los distintos años de estudio, al igual que para los datos globales, son registrados en la Tabla 3. Se observa que las variables Segunda Opción, Puntaje de Admisión, Icfes Ponderado y Sexo son consistentemente significativas para todos los años valorados.

Los resultados generados se obtuvieron aplicando el procedimiento Step en R, el cual incorpora tres estrategias de cálculo, a saber: la inclusión de variables una a una (Forward), la exclusión de variables una a la vez (Backward) y la que considera en cada paso una variable para inclusión o exclusión (Both) de un modelo completo. Esto arrojó en los tres casos la selección de todas las variables propuestas en el estudio. Además, para evaluar la bondad de ajuste del modelo, se calcularon los seudocoeficientes de determinación de Cox y Snell y Nagelkerke, con lo cual se obtuvo para el grupo total valores de 0,503 y 0,682 , respectivamente, por lo que el modelo global explicaría entre el 50,3\% y el 68,2\% de la variabilidad total en la decisión del estudiante de abandonar o permanecer en la institución. Asimismo, la prueba de Hosmer-Lemeshow arroja que el modelo global y los modelos por años, a excepción del 2011, se ajustan adecuadamente a los datos $(P>0,05)$.

Al realizar el análisis de los Odds ratios obtenidos en la Tabla 3, se observa que para la variable Segunda Opción se obtuvo un valor superior a uno $(2,99)$, lo que indica que un estudiante que ha ingresado al programa en la modalidad de segunda opción tiene un riesgo de abandonar sus estudios tres veces mayor que el estudiante que ingresa en primera opción. Los resultados son coherentes con los obtenidos por Rodríguez, Fita y Torrado (2004), quienes exponen que los estudiantes que escogen las carreras de su preferencia tienen mejores resultados que el resto de estudiantes. En el mismo sentido, Esteban, Bernardo, Tuero, Cerezo y Núñez (2016) comentan que el haber escogido la carrera 
Tabla 3. Odds ratios por años y para el global y estadisticos de bondad de ajuste

\begin{tabular}{|l|c|c|c|c|}
\hline \multirow{2}{*}{\multicolumn{1}{c|}{ Variables }} & \multicolumn{3}{c|}{ OR por años } \\
\cline { 2 - 5 } & $\mathbf{2 0 1 0}$ & $\mathbf{2 0 1 1}$ & $\mathbf{2 0 1 2}$ & Global \\
\hline Segunda Opción & $2,74^{* *}$ & $2,51^{* *}$ & $3,52^{* *}$ & $2,99^{* *}$ \\
\hline Acción Afirmativa & $0,60^{* *}$ & 0,92 & 0,95 & $0,82^{*}$ \\
\hline Méritos Académicos & 0,71 & 0,73 & $0,48^{* *}$ & $0,60^{* *}$ \\
\hline Icfes Ponderado & $0,96^{* *}$ & $0,97^{* *}$ & $0,97^{* *}$ & $0,97^{* *}$ \\
\hline Matrícula & 0,92 & $0,80^{* *}$ & 0,92 & $0,89^{* *}$ \\
\hline Sexo & $1,46^{* *}$ & $1,42^{* *}$ & $1,39^{* *}$ & $1,42^{* *}$ \\
\hline Tipo colegio & 1,06 & $1,40^{*}$ & 1,19 & $1,20^{* *}$ \\
\hline Edad ingreso & $1,03^{*}$ & 1,01 & $1,03^{*}$ & $1,03^{* *}$ \\
\hline Valor P Hosmer-Lemeshow & 0,220 & 0,036 & 0,200 & 0,318 \\
\hline R2 Cox \& Snell & 0,446 & 0,383 & 0,352 & 0,503 \\
\hline R2 Nagelkerke & 0,597 & 0,515 & 0,475 & 0,682 \\
\hline
\end{tabular}

${ }^{*} \mathrm{P}<0,05{ }^{* *} \mathrm{P}<0,01$.

Fuente: elaboración propia.

principalmente por vocación puede ser un factor protector del abandono, y agregan que los alumnos que eligen sus estudios por vocación dedican mayor tiempo a sus actividades escolares. Igualmente, Marín, Infante y Troyano (2000) afirman que el nivel de sacrificio es más liviano cuando la elección de los estudios universitarios se ha desarrollado durante un proceso reflexivo de autoconocimiento que pondera a la elección vocacional sobre otros motivos.

Análogamente, se registraron Odds ratios significativamente inferiores a uno $(\mathrm{P}<0,05)$ para las variables Acción Afirmativa, Méritos Académicos, Icfes Ponderado y Matrícula, todas ellas susceptibles de ser intervenidas institucionalmente. Ello indica que las mismas presentan un efecto protector de la permanencia estudiantil, por lo que los estudiantes que ingresaron accediendo a los cupos especiales por pertenecer a grupos vulnerables, por tener desempeños académicos destacados y los que obtuvieron mayores puntajes en las pruebas de admisión y pagaron un mayor monto de matrícula, presentaron un menor riesgo de deserción que aquellos clasificados en las categorías opuestas, resultados que confirman lo expuesto por Navarrete, Candia y Puchi (2013).

Para el tipo de admisión, se observa que las políticas adelantadas por la universidad con respecto al ofrecimiento de cupos especiales a estudiantes provenientes de poblaciones minoritarias y en condición vulnerable -que quedan en la categoría denominada Acción Afirmativa, al igual que los cupos ofrecidos por desempeños académicos destacados, categorizados bajo la denominación de Méritos Académicos- han permitido el acceso al sistema educativo universitario de jóvenes pertenecientes a grupos sociales desfavorecidos, atenuando con ello las desigualdades sociales, y se convierten en un factor protector, dado que registran una tasa de deserción más baja que la obtenida por los estudiantes regulares, lo que se evidencia más fuertemente entre los que acceden por méritos académicos.

A este respecto, Duarte, Galaz y Rosales (2005) comentan que un estudiante con buen rendimiento en el bachillerato cuenta con características, hábitos y estrategias de estudio y actitudes hacia el aprendizaje que le permiten desempeñarse acadé- 
micamente mejor en sus estudios universitarios, mientras que Rivera y Granobles (2012) plantean la hipótesis de que la capacidad y disposición para aprovechar las oportunidades pueden guardar alguna relación con la autoestima que les genera no solo ser los mejores bachilleres, sino que una universidad prestigiosa se lo reconozca. Por su parte, Geiser (2016) afirma que este tipo de programas representa una combinación poderosa de valores tanto igualitarios como meritocráticos.

En cuanto al valor pagado de la matrícula, los resultados obtenidos son coherentes con los hallazgos de otras investigaciones, y el riesgo de deserción disminuye conforme el ingreso del hogar se incrementa (CEDE, 2014). En el mismo sentido, Ishitani y Snider (2006) referencian que los estudiantes provenientes de hogares con mayores ingresos tienen mayor probabilidad de completar sus estudios, mientras que Sánchez y Otero (2012) exponen que las características socioeconómicas de la familia siguen siendo un determinante clave del rendimiento académico del estudiante y del ingreso esperado que tendrá en su vida adulta, lo que conlleva un círculo vicioso de reproducción de las desigualdades sociales.

Y con respecto a la última variable, correspondiente a la Edad que el estudiante tenía al ingreso a la universidad, se registró un Odds ratio significativamente superior a $1(\mathrm{P}<\mathrm{O}, \mathrm{O} 1)$, indicando con ello que a mayor edad se tiene un mayor riesgo relativo de deserción. En este sentido, un reporte del MEN (2008) afirma que el hecho de pasar de un rango alrededor de la media, es decir 17 años, a un rango de edad de 21 a 25 años incrementa el riesgo de deserción en un 39\%.

Teniendo en cuenta la alta heterogeneidad para las distintas áreas de conocimiento, lo que dificulta a su vez la búsqueda de regularidades y patrones en las mismas (Vélez y López, 2004), se realiza un análisis dentro de las distintas facultades, cuyos resultados muestran que el comportamiento ob- tenido efectivamente presenta matices diferentes, es decir, no todas las variables resultan ser significativas en todas las facultades, tal como queda evidenciado en la Tabla 4. Con excepción de la variable Sexo, que resulta ser significativa en todas las facultades, siendo las mujeres las que desertaron menos. Por su parte, la variable Ponderado Admisiones es significativa en todas las facultades, a excepción de la Facultad de Ciencias Agropecuarias.

Con relación a la variable Segunda Opción, es importante anotar que en las facultades de Ciencias Agropecuarias e Ingenierías no se registraron admitidos por segunda opción, y resulta ser significativa en las demás, a excepción de la Facultad de Ciencias de la Salud. En cuanto a las modalidades de admisión, se encuentran resultados particulares, siendo significativa Acción Afirmativa en las facultades de Ciencias Exactas y Naturales y de Ciencias Jurídicas y Sociales, mientras que Méritos Académicos resulta significativa solo en esta última. Por su parte, la variable Costo de Matrícula resulta significativa en Artes y Humanidades y en Ciencias para la Salud.

Llama la atención la baja correlación evidenciada entre la tasa de deserción y las distintas variables evaluadas para el caso de la facultad de Ciencias Agropecuarias, en donde solo el género resulta ser significativo. Y, particularmente, lo mostrado por la variable Ponderado de Admisiones, la cual no muestra asociación con la deserción, que sería lo esperado, lo que llevaría a cuestionar la idoneidad de los procesos de admisión actuales en dicha facultad.

Para evaluar el poder predictivo del modelo logístico calculado se acude a la generación de la Curva ROC de los datos pronosticados (Figura 2). El área bajo la curva obtenida fue de o,63, lo que refleja bajo poder predictivo, pero el contraste de aleatoriedad de la prueba -la cual plantea como hipótesis nula que la decisión pronosticada por el modelo es aleatoria, es decir, un área bajo la curva igual a o,5-es rechazado $(P=0,000)$, con un intervalo de confianza entre o,617 y o,644. Para la selección del punto de 
Tabla 4. Odds ratios estimados y valores $P$ de las variables por facultades

\begin{tabular}{|c|c|c|c|c|c|c|c|}
\hline \multirow{2}{*}{ Variables } & \multirow{2}{*}{ Estadisticos } & \multicolumn{6}{|c|}{ Facultades } \\
\hline & & $\mathbf{F}_{1}$ & $F_{2}$ & $F_{3}$ & $\mathrm{~F}_{4}$ & $\mathrm{~F}_{5}$ & F6 \\
\hline \multicolumn{2}{|c|}{ Deserción por facultades } & $38,20 \%$ & $43,50 \%$ & $45,30 \%$ & $46,80 \%$ & $42,50 \%$ & $32,90 \%$ \\
\hline \multirow{2}{*}{ Segunda opción } & O.R. & \multirow{2}{*}{ N.A. } & 0,36 & 0.18 & \multirow{2}{*}{ N.A. } & 0,22 & \multirow{2}{*}{ N.S. } \\
\hline & Valor P & & $\mathrm{O}$ & 0,028 & & $\mathrm{O}$ & \\
\hline \multirow{2}{*}{ Acción afirmativa } & O.R. & \multirow{2}{*}{ N.S. } & \multirow{2}{*}{ N.S. } & 0,53 & \multirow{2}{*}{ N.S. } & 0,63 & \multirow{2}{*}{ N.S. } \\
\hline & Valor P & & & 0,021 & & 0,019 & \\
\hline \multirow{2}{*}{$\begin{array}{c}\text { Méritos } \\
\text { académicos }\end{array}$} & O.R. & \multirow{2}{*}{ N.S. } & \multirow{2}{*}{ N.S. } & \multirow{2}{*}{ N.S. } & \multirow{2}{*}{ N.S. } & 0,49 & \multirow{2}{*}{ N.S. } \\
\hline & Valor P & & & & & 0,01 & \\
\hline \multirow{2}{*}{$\begin{array}{l}\text { Ponderado } \\
\text { admisiones }\end{array}$} & O.R. & \multirow{2}{*}{ N.S. } & 0,97 & 0,93 & 0,97 & 0,92 & 1,02 \\
\hline & Valor P & & $\mathrm{O}$ & 0.000 & 0,034 & $\mathrm{O}$ & 0,036 \\
\hline \multirow{2}{*}{ Costo matrícula } & O.R. & \multirow{2}{*}{ N.S. } & 0,86 & \multirow{2}{*}{ N.S. } & \multirow{2}{*}{ N.S. } & \multirow{2}{*}{ N.S. } & 0,81 \\
\hline & Valor P & & 0,041 & & & & 0,012 \\
\hline \multirow{2}{*}{ Sexo } & O.R. & 0,49 & 0.75 & 0,78 & 0,55 & 0,65 & 0,6 \\
\hline & Valor P & $\mathrm{O}$ & 0,018 & 0,079 & $\mathrm{O}$ & $\mathrm{O}$ & 0,001 \\
\hline \multirow{2}{*}{ Naturaleza colegio } & O.R. & \multirow{2}{*}{ N.S. } & 1,71 & \multirow{2}{*}{ N.S. } & \multirow{2}{*}{ N.S. } & \multirow{2}{*}{ N.S. } & \multirow{2}{*}{ N.S. } \\
\hline & Valor P & & $\mathrm{O}$ & & & & \\
\hline \multirow{2}{*}{ Edad ingreso } & O.R. & $A I C$ & 1,04 & 1,08 & $A I C$ & $\mathrm{NIC}$ & \\
\hline & Valor P & IV.S. & 0,054 & 0,001 & IV.S. & IV.S. & IV.S. \\
\hline Valor P Hosmer- & emeshow & 0,51 & 0,255 & 0,774 & 0,659 & 0,045 & 0,168 \\
\hline R2 Cox \& & nell & 0,444 & 0,475 & 0,474 & 0,429 & 0,51 & 0,425 \\
\hline R2 Nagelk & rke & 0,46 & 0,51 & 0,499 & 0,438 & 0,517 & 0,435 \\
\hline
\end{tabular}

F1: Facultad de Ciencias Agropecuarias; F2: Facultad de Artes y Humanidades; F3: Facultad de Ciencias Exactas y Naturales; F4: Facultad de Ingenierías; F5: Facultad de Ciencias Jurídicas y Sociales; F6: Facultad de Ciencias para la Salud; N.S: No Significativo (P > 0,1); N.A.: No Aplica. Fuente: elaboración propia.

corte óptimo se corre la función optimalCutoff del paquete InformationValue de $\mathrm{R}$, el cual genera un punto óptimo de 0,488 , es decir, probabilidades pronosticadas por el modelo por encima de tal valor serán categorizadas como indicadoras de desertores. De esta forma, la tasa de clasificación correcta obtenida fue de $61,2 \%$, con una sensibilidad de $59,4 \%$ y una especificidad de $62 \%$.

Es de anotar que, dado que la problemática de deserción atañe a un fenómeno complejo y multifactorial (Tinto, 1975; MEN, 2008), el nivel predictivo de los procesos de admisión es relativamente modesto y, así mismo, la capacidad de predecir los resultados en la universidad es relativamente limitada (Geiser,
Figura 2. Curva ROC del modelo logístico obtenido

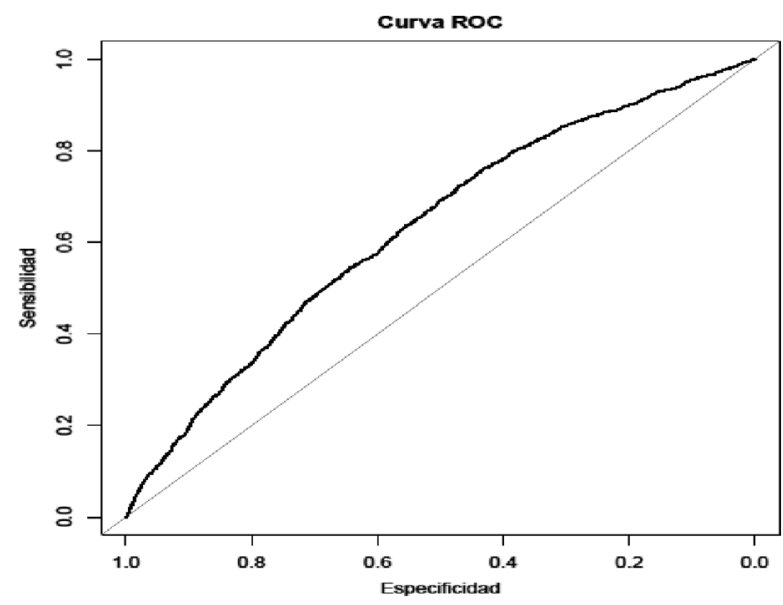

Fuente: elaboración propia. 
2016), por lo que los resultados aquí reportados no distan significativamente de otros estudios afines (Barahona, 2014).

Finalmente, el cálculo de las ponderaciones óptimas para las distintas áreas evaluadas en las pruebas de Estado en función de su correlación con las notas promedio en la carrera arroja los resultados que se muestran en la Tabla 5. En la misma se resaltan aquellas áreas evaluadas que registran mayores ponderaciones en los respectivos programas.

Es de anotar que los cálculos solo se realizaron para tres programas de la Facultad de Ciencias Exactas y Naturales, dado que se exponen como una posible estrategia de análisis para abordar la evaluación de las ponderaciones asignadas. Igualmente es importante precisar que los resultados obtenidos son el producto de una propuesta de optimización, entre muchas otras que se podrían plantear, por lo que algunos resultados obtenidos pueden no parecer razonables a la luz de su afinidad con el programa, pero son los que el algoritmo arroja en su procesamiento numérico y sirven como punto de partida para la discusión sobre la pertinencia a los posibles cambios a las ponderaciones existentes en la actualidad.
Se resalta, entre los datos obtenidos, que para el programa de Geología las más altas ponderaciones se obtienen en las áreas de Matemáticas y Química, que son precisamente aquellas en las cuales se les da mayor peso en la actualidad. Por el contrario, para el programa de Biología la ponderación más alta obtenida resulta ser en la prueba de Filosofía, área que no es valorada en la ponderación actual. En igual sentido, para el programa de Biología y Química se registran altas ponderaciones en Lenguaje y, de forma poco consistente, en Geografía.

\section{Conclusiones y recomendaciones}

Aunque el abandono estudiantil universitario ha sido una temática ampliamente estudiada a nivel mundial, son pocos los estudios que la abordan con base en el análisis de la incidencia que tienen los mecanismos de selección de ingreso a la universidad sobre la deserción. Más aún, si se tiene en cuenta que los mismos atañen a factores que se pueden modificar por acciones de gestión institucional y que proporcionan una vía para el diagnóstico temprano de los estudiantes que ingresan a la universidad.

Tabla 5. Ponderaciones por normativa y las generadas en el proceso de optimización

\begin{tabular}{|c|c|c|c|c|c|c|}
\hline \multirow[t]{2}{*}{ Área } & \multicolumn{2}{|c|}{ Biología y Química } & \multicolumn{2}{|c|}{ Biología } & \multicolumn{2}{|c|}{ Geología } \\
\hline & Actual & Óptimo & Actual & Óptimo & Actual & Óptimo \\
\hline Biología & $15 \%$ & $2,70 \%$ & $20 \%$ & $7,10 \%$ & ०\% & ०\% \\
\hline Filosofía & $10 \%$ & ०\% & ०\% & $47,90 \%$ & ०\% & $11,10 \%$ \\
\hline Historia & $5 \%$ & ०\% & $5 \%$ & $3,60 \%$ & ०\% & ०\% \\
\hline Lenguaje & $15 \%$ & $39,20 \%$ & $25 \%$ & $8,10 \%$ & O\% & $7,80 \%$ \\
\hline Matemáticas & $20 \%$ & $7,20 \%$ & $25 \%$ & $9,10 \%$ & $30 \%$ & $35 \%$ \\
\hline Física & $15 \%$ & ०\% & $10 \%$ & $0,30 \%$ & $20 \%$ & $4,90 \%$ \\
\hline Química & $15 \%$ & $7,30 \%$ & $10 \%$ & $23,40 \%$ & $35 \%$ & $27,80 \%$ \\
\hline Geografía & $5 \%$ & $43,60 \%$ & $5 \%$ & $0,40 \%$ & $15 \%$ & $12,00 \%$ \\
\hline Interdisciplinar & ०\% & ०\% & ०\% & $0,10 \%$ & ०\% & $1,40 \%$ \\
\hline Coeficiente de correlación & 0,082 & 0,173 & 0,096 & 0,158 & 0,236 & 0,269 \\
\hline Incremento & \multicolumn{2}{|c|}{0,091} & \multicolumn{2}{|c|}{0,062} & \multicolumn{2}{|c|}{0,033} \\
\hline
\end{tabular}

Fuente: elaboración propia. 
Entre los resultados a destacar, se observa que las variables referentes a los procesos de admisión a la universidad muestran una relación significativa con el riesgo de deserción. Puntualmente, se encuentra que el ingreso a un programa de la universidad mediante la modalidad de segunda opción está asociado a un alto riesgo de deserción, lo que lleva a pensar en la necesidad de reevaluar dicha opción de admisión. En igual sentido, se halla que, como era de esperarse, el puntaje de admisión muestra una relación altamente significativa con el riesgo de abandono y que el nivel socioeconómico del estudiante, expresado en costo de matrícula, está directamente relacionado con la deserción y, a pagos de matrículas mayores, el riesgo desciende.

En cuanto a las variables relacionadas con el tipo de admisión, se encuentra que las estrategias gestionadas por la institución, que permiten ofertar cupos especiales a estudiantes provenientes de poblaciones vulnerables, y las que hacen reconocimiento a desempeños meritorios en la secundaria, han mostrado ser un factor protector del abandono estudiantil, ya que los que acceden a tales programas presentan un riesgo de deserción inferior a los que lo hacen de forma regular. Frente a las acciones afirmativas, es necesario resaltar que las desigualdades sociales tienen normalmente un origen en acciones $\mathrm{u}$ omisiones por parte del Estado, que a su vez están legitimadas por actitudes y disposiciones culturalmente aprendidas y reproducidas (Galtung,
1990). Por ello es necesario mantener las políticas de admisión y acceso de los grupos étnicos y de aquellos que hacen parte de comunidades cuyas condiciones sociales no les permiten competir en igualdad de condiciones en el ingreso a la Universidad.

Es importante plantear la articulación de los niveles de educación media y universitaria como mecanismo de apoyo y fortalecimiento de los procesos de orientación vocacional para que los estudiantes hagan una elección de carrera apoyados en un mejor conocimiento de sus aptitudes y motivaciones. Con relación al proceso de ponderaciones, se sugiere la revisión de los porcentajes asignados a las áreas predefinidas para la selección de los estudiantes y la consideración de áreas no contempladas en dicho proceso. Finalmente, se recomienda la realización de trabajos que acudan a la obtención de datos primarios, orientadas al diseño y aplicación de estrategias que permitan evidenciar de forma más directa las relaciones identificadas en esta investigación.

\section{Agradecimientos}

A la instituciones educativas de la ciudad de Manizales: Liceo Arquidiocesano Nuestra Señora, Liceo Isabel la Católica, Instituto Tecnológico, Instituto Universitario de Caldas; a la Vicerrectoría de Investigaciones de la Universidad de Caldas y al programa Reconstrucción del tejido social en zonas de posconflicto en Colombia, con código de la Vicerrectoría de Investigaciones y Postgrados 2012917. 


\section{Referencias}

Barragán, D., Patiño, L. (2013). Elementos para la comprensión del fenómeno de la deserción universitaria en Colombia. Más allá de las mediciones. Cuadernos Latinoamericanos de Administración, 9(16), 55-66. DOI: 10.18270/cuaderlam.vgi16.1248

Bean, J. P. (1990). Why students leave: Insights from research. En The strategic management of college enrollments (pp. 147-169). San Francisco: Jossey-Bass.

Blanco Suárez, J. O. (2014). Educación superior, crédito educativo y equidad en Colombia 1992-2011. Diálogos de Saberes, 40, 139-155. DOI: 10.18041/0124-0021/dialogos.40.214

Cabrera, A., M. Castañeda, A. Nora y D. Hengstler (1992). The Convergence between two theories of college persistence. Journal of Higher Education, 63(2), 143-164. 10.2307/1982157

Cabrera, A.; Pérez, P. y López, L. (2015). Evolución de perspectivas en el estudio de la retención universitaria en los Estados Unidos: bases conceptuales y puntos de inflexión. En P. Figuera (ed.), Persistir con éxito en la universidad: de la investigación a la acción (pp. 15-40). Barcelona: Laertes.

Cabrera, L., Tomás, J. T., González, M. y Álvarez, P. (2006). Un estudio transversal retrospectivo sobre la prolongación y abandono de estudios universitarios. Revista Electrónica de Investigación y Evaluación Educativa, 12(1), 105-127. DOI: 10.7203/relieve.12.1.4241

Castellanos, J. M., Correa, B. L.y Loaiza, M. O. (2006). Espirales de humo. El acceso de estudiantes de grupos étnicos en la Universidad. Facultad de Ciencias Jurídicas y Sociales, Grupo de investigación Comunicación, Cultura y Sociedad. Manizales: Universidad de Caldas.

CEDE - Centro de Estudios sobre Desarrollo Económico (2014). Determinantes de la deserción. Informe de la Facultad de Economía, Universidad de los Andes. Recuperado de: https://www.mineducacion.gov.co/sistemasdeinformacion/1735/articles-254702_Informe_determinantes_desercion.pdf

De Vries, W.; León Arenas, P., Romero Muñoz, J. F. y Hernández Saldaña, I. (2011). ¿Desertores o decepcionados? Distintas causas para abandonar los estudios universitarios. Revista de la Educación Superior, 40(160), 2949. Recuperado de http://publicaciones.anuies.mx/pdfs/revista/Revista16o_S1A3ES.pdf

Donoso, S. y Schiefelbein, E. (2007). Análisis de los modelos explicativos de retención de estudiantes en la universidad: una visión desde la desigualdad social. Estudios Pedagógicos, 33(1), 7-27. Recuperado de https:// www.redaly c.org/articulo.oa?id=173514133001

Duarte, M., Galaz, J. y Rosales, J. (2005). Predictores de desempeño académico en una cohorte al finalizar el $2^{\text {do }}$ y $5^{\text {to }}$ semestre en una universidad pública estatal. Recuperado de: http://www.comie.org.mx/congreso/ memoriaelectronica/vog/ponencias/at16/PRE1178912891.pdf 
Escanés, G., Herrero, V., Merlino, A. y Ayllón, S. (2014). Deserción en educación a distancia: factores asociados a la elección de modalidad como desencadenantes del abandono universitario. Virtualidad, Educación y Ciencia, 9(5), 45-55. Recuperado de https://revistas.unc.edu.ar/index.php/vesc/article/view/9549

Esteban, M., Bernardo, A., Tuero, E., Cerezo, R. y Núñez, J. (2016). El contexto sí importa: identificación de relaciones entre el abandono de titulación y variables contextuales. European Journal of Education and Psychology, 9, 79-88. DOI: 10.1016/j.ejeps.2015.06.001

Esteban, M., Bernardo, A., Tuero, E., Cervero, A. y Casanova, J. (2017). Variables influyentes en progreso académico y permanencia en la universidad. European Journal of Education and Psychology, 10, 75-81. DOI: 10.1016/j. ejeps.2017.07.003

Fonseca, G. y García, F. (2016). Permanencia y abandono de estudios en estudiantes universitarios: un análisis desde la teoría organizacional. Revista de Educación Superior, 45(179), 25-39. DOI:10.1016/j.resu.2016.06.004

Galtung, J. (1990). Cultural violence. Journal of Peace Research, 27(3), 291-305. Recuperado de https://www.jstor. org/stable/423472

Garbanzo, G. (2007). Factores asociados al rendimiento académico en estudiantes universitarios, una reflexión desde la calidad de la educación superior pública. Revista Educación, 31(1), 43-63. DOI: 10.15517/revedu. v3111.1252

Geiser, S. (2016). Medición y evaluación para los procesos de admisión de la educación superior: hallazgos desde California. Revista de Investigación Educacional Latinoamericana, 53(1), 1-18. DOI: 10.7764/PEL.53.1.2016.7

Gómez, V.y Celis, J. (2009). Crédito educativo, acciones afirmativas y equidad social en la educación superior en Colombia. Revista de Estudios Sociales, 30, 106-117. DOI: 10.7440/res33.2009.09

Green, W. (2012). Econometric analysis (7 ed.). Pearson.

Hoare, A. y Johnston, R. (2011). Widening participation through admissions policy- a British case study of school and university performance. Studies in Higher Education, 36(1), 21-41. DOI: 10.1080/03075070903414297

Ishitani, T. y Snider, K. (2006). Longitudinal Effects of College Preparation Programs on College Retention. Association for Institutional Research, 9(3), 1-10. Recuperado de https://eric.ed.gov/?id=ED504377

Khuong, H. (2014). Evaluation of a conceptual model of student retention at a public urban commuter university. e-Commons. Chicago: Loyola Univerty Chicago. (Dissertations 1092). Recuperado de: http://ecommons. luc.edu/luc_diss/1092

Makransky, G., Havmose, P., Vang, M., Andersen, T.y Nielsen, T. (2016). The predictive validity of using admissions testing and multiple mini-interviews in undergraduate university admissions. Higher Education Research \& Development, 36(5). DOI: 10.1080/07294360.2016.1263832 
Marín, M., Infante, E. y Troyano, Y. (2000). El fracaso académico en la universidad: aspectos motivacionales e intereses profesionales. Revista Latinoamericana de Psicología, 32(3), 505-517. Recuperado de http://personal.us.es/einfante/uploads/PUBLICACIONES/Fracaso\%2Oacad\%C3\%Agmico\%20Mar\%C3\%ADn-InfanteTroyanozooo.pdf

Melguizo, T., Sánchez, F.y Jaime, H. (2011). The association between financial aid availability and the college dropout rates in Colombia. Higher Education, 62, 231-247. DOI: 10.1007/s10734-010-9385-8

MEN - Ministerio de Educación Nacional (2008). Análisis de determinantes de la deserción en la educación superior colombiana con base en el Spadies. Bogotá.

MEN - Ministerio de Educación Nacional (2009). Deserción estudiantil en la educación superior colombiana. Metodología de seguimiento, diagnóstico y elementos para su prevención. Recuperado de: https://www. mineducacion.gov.co/sistemasdeinformacion/1735/articles-254702_libro_desercion.pdf

MEN - Ministerio de Educación Nacional de Colombia (2010). 778 mil millones de pesos le costó a Colombia la deserción universitaria en 2009. Observatorio, 23 de noviembre. Recuperado de: https://www.mineducacion.gov.co/observatorio/1722/article-256223.html

MEN - Ministerio de Educación Nacional de Colombia (2016). La educación en Colombia. Bogotá: MEN / OECD. Recuperado de: https://www.mineducacion.gov.co/1759/articles-356787_recurso_1.pdf

MEN - Ministerio de Educación Nacional de Colombia (2016). Estadísticas Deserción y Graduación 2015. Recuperado de: https://www.mineducacion.gov.co/sistemasdeinformacion/1735/articles-357549_recurso_3.pdf

Morón-Cárdenas, J., Pedrozo-Martínez, A. y Torres-Cabrera, M. (2013). Acciones afirmativas como mecanismo de inclusión social en la educación superior pública: el caso de la Región Caribe colombiana. Clío América, 7(14),110-119. Recuperado de http://revistas.unimagdalena.edu.co/index.php/clioamerica/article/view/756

Mullen, K., Ardia, D., Gil, D., Windover, D. y Cline, J. (2011). DEoptim: An R Package for Global Optimization by Differential Evolution. Journal of Statistical Software, 40(6), 1-26. DOI: 10.18637/jss.v040.io6

Navarrete, S., Candia, R. y Puchi, R. (2013). Factores asociados a la deserción/retención de los estudiantes mapuche de la Universidad de la Frontera e incidencia de los programas de apoyo académico. Calidad en la Educación, 39, 43-80. DOI: 10.4067/S0718-45652013000200003

Rivera, B. y Granobles, J. (2012). Análisis de políticas compensatorias para la equidad en el acceso a la educación superior: el caso de la Universidad de Caldas. El Pensamiento Universitario, 23, 81-91. Bogotá: Asociación Colombiana de Universidades.

Rodríguez, S., Fita, E. y Torrado, M. (2004). El rendimiento académico en la transición Secundaria-Universidad. Revista de Educación, 334, 391-414. Recuperado de http://www.revistaeducacion.mec.es/re334_22.htm

Ruiz, F.y Rodríguez, M. (2011). Factores motivacionales que orientan la decisión de ingresar a la Licenciatura en Biología y Química de la Universidad de Caldas, Colombia. Revista Latinoamericana de Estudios Educativos, 7(2), 11-35. Recuperado de http://www.redaly c.org/articulo.oa?id=134125454003 
ISSN 0123-1294 | e-ISSN 2027-5358 | Educ.Educ. Vol. 22. No.1 | Enero-abril de 2019 | pp. 81-100.

Universidad de La Sabana | Facultad de Educación

Sánchez, A. y Otero, A. (2012). Educación y reproducción de la desigualdad en Colombia. Reportes del Emisor, Investigación e Información Económica No. 154. Bogotá: Banco de la República.

Sánchez, F., Q-uirós, M., Reverón, C. y Rodríguez, A. (2002). Equidad social en el acceso y permanencia en la Universidad Pública determinantes y factores asociados. Bogotá: Universidad de los Andes - Centro de Estudios Económicos.

Silva, M. (2011). El primer año universitario. Un tramo crítico para el éxito académico. Perfiles Educativos, 33, número especial, 102-114. Recuperado de http://www.redaly c.org/articulo.oa?id=13221258010

Tapasco, O., Ruiz, F. y Osorio, D. (2016). Estudio del poder predictivo del puntaje de admisión sobre el desempeño académico universitario. Revista Latinoamericana de Estudios Educativos, 12(2), 148-165. Recuperado de http://latinoamericana.ucaldas.edu.co/downloads/Latinoamericana12(2)_8.pdf

Tinto, V. (1975). Dropout from Higher Education: A Theoretical Synthesis of Recent Research. Review of Educational Research, 45(1), 89. DOI: 10.2307/1170024

Tinto, V. (1993). Leaving college: rethinking the causes and cures of student attrition. Chicago: University of Chicago Press.

Tonconi, Q. (2010). Factores que influyen en el rendimiento académico y la deserción de los estudiantes de la facultad de ingeniería económica de la una-puno, periodo 2009. Revista de educación y desarrollo, 2(11), 2010. Recuperado de http://www.eumed.net/rev/ced/11/jtq.htm

Torres-Guevara, L. E. (2012). Retención estudiantil en la educación superior. Bogotá: Pontificia Universidad Javeriana.

Vélez, A. y López, D. (2004). Estrategias para vencer la deserción universitaria. Educación y Educadores, 7, 177-203. Recuperado de http://educacionyeducadores.unisabana.edu.co/index.php/eye/article/view/555

Vergara-Díaz, G. y Peredo-López, H. (2017). Relación del desempeño académico de estudiantes de primer año de universidad en Chile y los instrumentos de selección para su ingreso. Revista Educación, 41(2). DOI: 10.15517/revedu.v41i2.21514

Weidman, J. (1989). Undergraduate socialization: a conceptual approach. En J. Smart (ed.), Higher education: Handbook of theory and research (vol. 5). Nueva York: Agathon. 